\section{$-$ The Dippy Dentist}

H. S. Brand of the Academic Centre for Dentistry Amsterdam, and movie buff, continues his new series of columns on films featuring dentists with The Dippy Dentist (1920), directed by Alf Goulding

During the Prohibition era, two dental offices share a floor of an office building with a beauty parlour. One of the two dentists sees reluctantly that the waiting room of the other dentist is full of patients, while he has no patients at all.

He sneaks into his competitor's office, and with the smell of a bottle of liquor, he lures all the patients into his own office, including the patient who already had taken place in the dental chair.

After arrival in his own dental practice, the dentist roughly lifts the patient into the dental chair. The patient points to a tooth in the lower front, and subsequently the dentist tries to sedate the patient with nitrous oxide twice. Since that does not work, he hits him on his head with a wooden hammer.

Just when the dentist wants to extract the tooth, the beauty specialist Fifi enters. Fifi holds a hand against her cheek, saying: 'Doctor, it hurts like I had a mouthful of firecrackers!'.

Distracted by her, the dentist destroys the patient's glasses and moustache with the extraction tongs. The dentist asks Fifi to take a seat in the waiting room and continues to pull the tooth in a rough way.

The patient is being dragged around the entire dental office and eventually falls out of the window, a few floors down on the sidewalk. When the patient complains to the police officer, the dentist calls from the window: 'Hold him, officer! He jumped his bill'.

Now Fifi takes the empty place in the dental chair. After she has been sedated with nitrous oxide, the dentist starts kissing her repeatedly. At that moment the police officer, the boyfriend of Fifi, comes in. He chases the dentist through the building and finally manages to arrest him.

\title{
Whole NHS team to ensure oral health is central to child care
}

The whole clinical team at a large hospital in London is being trained to ensure that oral health is a central part of the care being provided to children there.

Paediatric dental consultant Urshla Devalia and specialist dental nurse Claire Fletcher are helping to implement a pioneering new programme called Mini Mouthcare Matters (Mini MCM) at Great Ormond Street Hospital (GOSH) in London in what is hoped will become a national initiative.

Under the programme, Devalia and Fletcher will train nurses, healthcare assistants and doctors working at the hospital in the importance of oral healthcare, stressing that a simple toothbrush can be critical to health and well-being of seriously ill children.

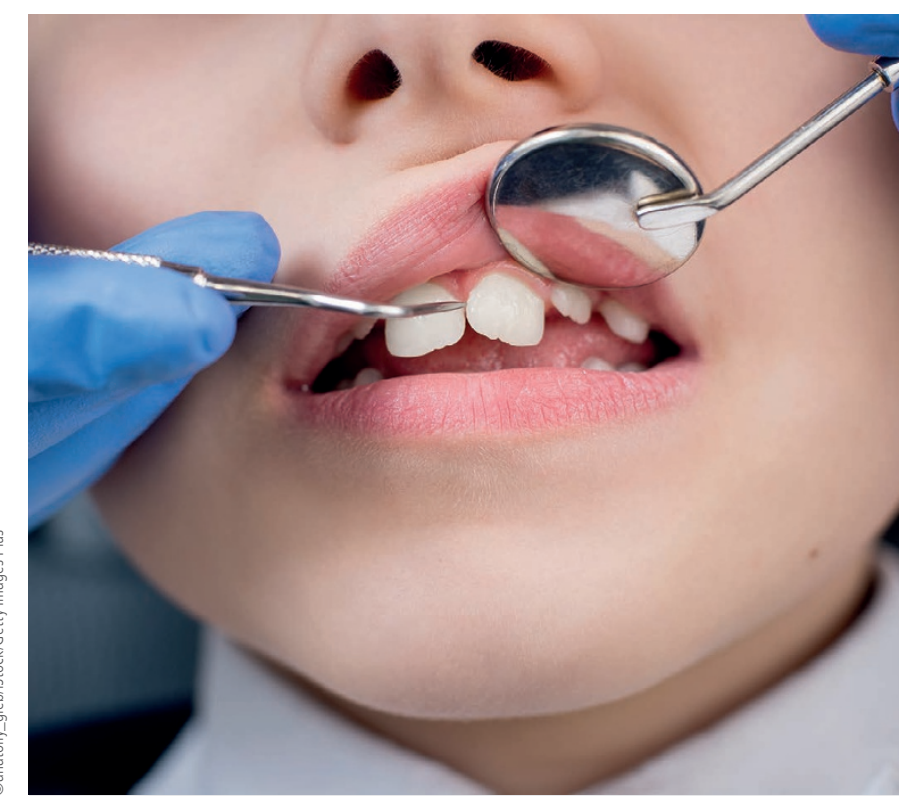

In future, staff on paediatric wards will integrate dental health into the overall care they offer, meaning that they will, for example, ask young patients - or their parents - if they have brought in a toothbrush for their overnight stay and when they last saw a dentist.

The programme, funded by Health Education England and GOSH will be working in collaboration with Birmingham Children's Hospital, Royal Manchester Children's Hospital and University College London Hospitals with a view to rolling the programme out nationally in 2019.

Devalia, a member of the British Society of Paediatric Dentistry's (BSPD) executive committee, said her aim was to empower all healthcare professionals working in hospitals with paediatric wards to incorporate oral health into the care routine.

Devalia said: 'Our young patients are already struggling with complex and serious conditions. It's vital that there is no risk of an infection in the mouth which could cause complications in their treatment. By integrating dental hygiene into overall care, health professionals can take every opportunity to highlight how important a healthy mouth and teeth are to general well-being.'

To support ward nurses and healthcare assistants, the initiative will be developing:

- An oral health policy and tooth brushing chart, fully adaptable to any hospital trust

- An oral health screening tool to identify patients who may be at a high risk of developing oral problems

- A training package to encourage all nursing, medical and health care professionals to 'lift the lip' and identify common oral health/ dental conditions

- A range of tools applicable to paediatric in-patients that can be used by all hospitals hoping to join Mini MCM, including posters, booklets and baseline audit tools. 